\title{
Galanin Receptor 1 Plays an Antinociceptive Effect via Inhibiting PKA Activation in the Nucleus Accumbens of Rats With Neuropathic Pain
}

\author{
Ying ZHANG ${ }^{* *}$, Yi GAO ${ }^{2 *}$, Chong-Yang $\mathrm{LI}^{3}$, Wei DONG ${ }^{4}$, Yan DONG ${ }^{1}$, Meng-Nan LI ${ }^{1}$, \\ Ya-Nan LIU ${ }^{1}$, Shi-Lian XU ${ }^{1}$ \\ * These authors contributed equally to this work.
}

${ }^{1}$ Department of Physiology, School of Basic Medicine, Kunming Medical University, Kunming, China, ${ }^{2}$ Department of Colorectal Cancer Surgery, Third Affiliated Hospital, Kunming Medical University, Kunming, China, ${ }^{3}$ Department of Tumor, Fourth Affiliated Hospital, Kunming Medical University, Kunming, China, ${ }^{4}$ Department of Orthopedics, Third Affiliated Hospital, Yunnan Traditional Chinese Medical College, Kunming, China

Received May 9, 2018

Accepted January 29, 2019

Epub Ahead of Print March 22, 2019

\section{Summary}

Galanin and galanin receptors (GalRs) have been reported to be involved in the transmission and modulation of nociceptive information in the central nervous system (CNS). However, the underlying mechanism of the antinociception of GalRs in neuropathic pain remains unclear. This study investigated the antinociception induced by galanin receptor 1 (GalR1) via protein kinase A (PKA) signaling pathway in the nucleus accumbens (NAc) of rats with neuropathic pain. A mononeuropathy model was replicated by ligation of the left sciatic nerve, following which the expression of phospho-PKA (p-PKA) in the NAc were markedly up-regulated at $14^{\text {th }}$ and $28^{\text {th }}$ day after ligation of sciatic nerve, and p-PKA expression was down-regulated by intra-NAc injection of GalR1 agonist M617, but the GalR1 antagonist M35 did not have an effect. We also found that M35 in the NAC blocked the M617-induced increase in the hind paw withdrawal latencies (HWLs) of rats with mononeuropathy, but M35 alone had no effect on HWLs, and PKA inhibitor H-89 attenuated the M617-induced an increase in the HWLs. These results suggested that GalR1 induced an antinociception via inhibiting PKA activation, implying that GalR agonists may be potential and potent therapeutic options to treat chronic neuropathic pain.

\section{Key words}

Antinociception - Galanin receptor 1 - Protein kinase A • Neuropathic pain $\bullet$ Nucleus accumbens

\section{Corresponding author}

S.-L. Xu, Department of Physiology, School of Basic Medicine, Kunming Medical University, Kunming 650500, China. E-mail: shilianxu@126.com

\section{Introduction}

Neuropathic pain caused by damage or disease is very common in the clinic, but its complex pathogenesis has hampered the effective treatment of neuropathic pain (Costigan et al. 2009). The development of current therapeutics that have been focusing on neuronal targets, including drugs such as opioid analgesics, NMDA receptor antagonists and sodium channel blockers (Dworkin et al. 2003). These drugs produce a brief pain relief in some patients, however, the side effects of these drugs also are notably, such as sedation, nausea and dizziness, as well as development of analgesic tolerance and addiction after opioid treatment, these side effects have greatly limited their universal use (Dworkin and Turk 2011, Ho and Siau 2009). Therefore, we need to find a safer and more effective analgesic.

Galanin was first separated from the porcine intestine. The function of galanin has been linked to the activation of specific galanin receptors (GalRs). Three galanin receptors (GalR1-3) have been identified 
(Branchek et al. 2000). The signaling pathways of three GalRs are essentially different (Lang et al. 2015). Our earlier research demonstrated that M617, a specific GalR1 agonist, has an antinociceptive effect in rats with neuropathic pain (Duan et al. 2015), indicating that GalR1 activation induced an antinociception effect. However, the signaling pathways mechanism of the antinociception of GalR1 in neuropathic pain is unclear.

Intracellular protein kinases play a significant role in the processing and development of chronic pain. Some studies have demonstrated that protein kinase A (PKA) activation plays an important functional role in the processing of nociceptive information (Hang et al. 2012, Hang et al. 2013, Zhu et al. 2014). This study investigated the antinociception induced by GalR1 via PKA signaling pathway in the rats with neuropathic pain.

The nucleus accumbens (NAc) is divided into two regions: the core and shell, which are known to be the brain regions involved in pain modulation (Salgado and Kaplitt 2015). Gear et al. (2011) reported that NAc acts an important effect in mediating the suppression of tonic or persistent pain. Infusion of $\mathrm{N}$-acetylaspartylglutamate into the NAc significantly attenuated the pain induced by the activation of sensory nerves through optical stimulation (Watanabe et al. 2018). Previous studies in our laboratory have demonstrated that the expression of GalR1 was up-regulated in the NAc of rats with inflammation or sciatic nerve ligation, intra-NAc injection of galanin or GalR1 agonist M617 induced dose-dependent increase in hind paw withdrawal latencies (HWLs) to noxious thermal and mechanical stimulations in rats, suggesting galanin and GalR1 are involved in antinociceptive effects in the NAc of rats (Xu et al. 2012, Duan et al. 2015, Yang et al. 2015). However, the interaction mechanism is unclear between galanin and GalRs as well as downstream signal molecule in pain modulation in the NAc. In this study, we intended to investigate the involvement of PKA in the GalR1-induced antinociception in the NAc of rats with neuropathic pain.

\section{Methods}

\section{Animals}

Male Sprague-Dawley rats (weighing 180-250 g) were obtained from the experimental Animal Center of Kunming Medical University (Kunming, Yunnan, China). Rats were housed in cages with water and food available ad libitum in a temperature-controlled $\left(22 \pm 1^{\circ} \mathrm{C}\right)$ room with a normal day/night cycle. Experimental procedures were in strict accordance with the European Guidelines on Laboratory Animal Care.

\section{Chemicals}

M617 (galanin(1-13)-Gln(14)-bradykinin(29)amide), M35 (galanin(1-13)-bradykinin (2-9)amide) and $\mathrm{H}-89$ hydrochloride $\left(\mathrm{C}_{20} \mathrm{H}_{20} \mathrm{BrN}_{3} \mathrm{O}_{2} \mathrm{~S} .2 \mathrm{HC}\right)$ were purchased from Sigma-Aldrich Inc. (St. Louis, MO, USA). M617 and M35 were dissolved in physiologic saline, $\mathrm{H}-89$ was dissolved in $1 \%$ dimethyl sulfoxide (DMSO). The solutions were injected into the NAc at a total volume of one microliter.

\section{Neuropathic pain animal model}

The sciatic nerve ligation procedure for eliciting neuropathic pain was carried out as described previously (Bennett and Xie 1988). Briefly, a rat was anaesthetized intraperitoneally with sodium pentobarbital $(50 \mathrm{mg} / \mathrm{kg})$, and the left sciatic nerve was exposed for approximately $1 \mathrm{~cm}$ at the middle of thigh. Then, a total of 4 knots approximately $1 \mathrm{~mm}$ apart were loosely ligated with 4.0 chromic gut sutures.

In the sham control group, the same operation protocols were used without ligation of the sciatic nerve. In both groups, the contralateral limb remained un-operated.

\section{Behavioral test}

The hind paw withdrawal latency (HWL) responses to noxious mechanical and thermal stimuli were tested as previously described (Xu et al. 2012, Yang et al. 2015, Zhang et al. 2017). A hot plate (Type YLS-6B, China) was used to test HWL to noxious thermal stimulation. The temperature of the hot plate was kept within $52 \pm 0.2{ }^{\circ} \mathrm{C}$. The radiant heat source was focused on the planta of the hind paw, and the HWL was recorded.

The Randall Selitto Test (Ugo Basile, Type 37215 , Italy) was used to evaluate the HWL to noxious mechanical stimulation. A pusher was applied to the dorsal surface of the rat's hind paw at a loading rate of $30 \mathrm{~g} / \mathrm{s}$, and the threshold required to initiate the struggle response was used as the HWL to mechanical stimulation.

Rats were individually acclimated to thermal and mechanical stimulation for 4-5 days before sciatic nerve ligation, and the HWLs were normally between 3-6 s. The HWL was detected three times before intra-NAc 
injection to get an average value as the baseline HWL. The HWL was detected at $5^{\text {th }}, 10^{\text {th }}, 15^{\text {th }}, 20^{\text {th }}, 30^{\text {th }}, 45^{\text {th }}$ and $60^{\text {th }}$ min after intra-NAc injection, and each HWL was recorded as a percentage change from the baseline HWL.

\section{Intra-NAc injection}

The rat was anesthetized using sodium pentobarbital $(50 \mathrm{mg} / \mathrm{kg}$, intraperitoneal injection) and placed in a stereotaxic instrument. A stainless steel guide cannula with outer diameter of $0.8 \mathrm{~mm}$ was penetrated to the NAc (anterior to Bregma: $1.7 \mathrm{~mm}$; left or right to midline: $1.6 \mathrm{~mm}$; ventral: $7.0 \mathrm{~mm}$ ).

Rats recovered from the operation for 3 days. A stainless steel needle with outer diameter of $0.4 \mathrm{~mm}$ was inserted into guide cannula and deepened $1.5 \mathrm{~mm}$ to the cannula. One microliter of solution was injected into the NAc.

\section{Western blot assay}

Rats were anaesthetized with $5 \%$ isoflurane and euthanized. Then, the NAc tissue was promptly excised. The Western blot assay procedure was performed as described previously (Duan et al. 2015, Yang et al. 2015). Primary antibodies against the following proteins were used: total-PKA (t-PKA, rabbit polyclonal antiPKA alpha+beta, 1:200, Abcam), and phospho-PKA (p-PKA, rabbit polyclonal anti-PKA alpha+beta phospho, 1:300, Abcam). The expression of glyceraldehyde-3phosphate dehydrogenase (GAPDH, rabbit monoclonal, $1: 5,000$, CellSignal) was also determined as an internal control. The bands were exposed to Fuji Medical X-Ray Film (Fuji Photo Film Co., Ltd, Karagawa, Japan) and the relative band density was detected by Image J software. The Western blot of the protein samples from the NAc of each experimental rat was repeated at least 3 times.

\section{Statistical analysis}

At the end of the experiments, the location of the needle tip was confirmed. As shown in Figure 1, only the results from rats whose needle tip was within the NAc were used for statistical analysis. Data were expressed as the mean \pm S.E.M. and analysed using GraphPad Prism 5 software. The statistical analyses were calculated using one-way analysis of variance test (ANOVA) followed by Tukey's multiple comparison tests or two-way repeated measure ANOVA followed by Bonferroni post-tests or Student's two tailed t-test. The $\mathrm{P}<0.05$ were considered to indicate a significant difference.

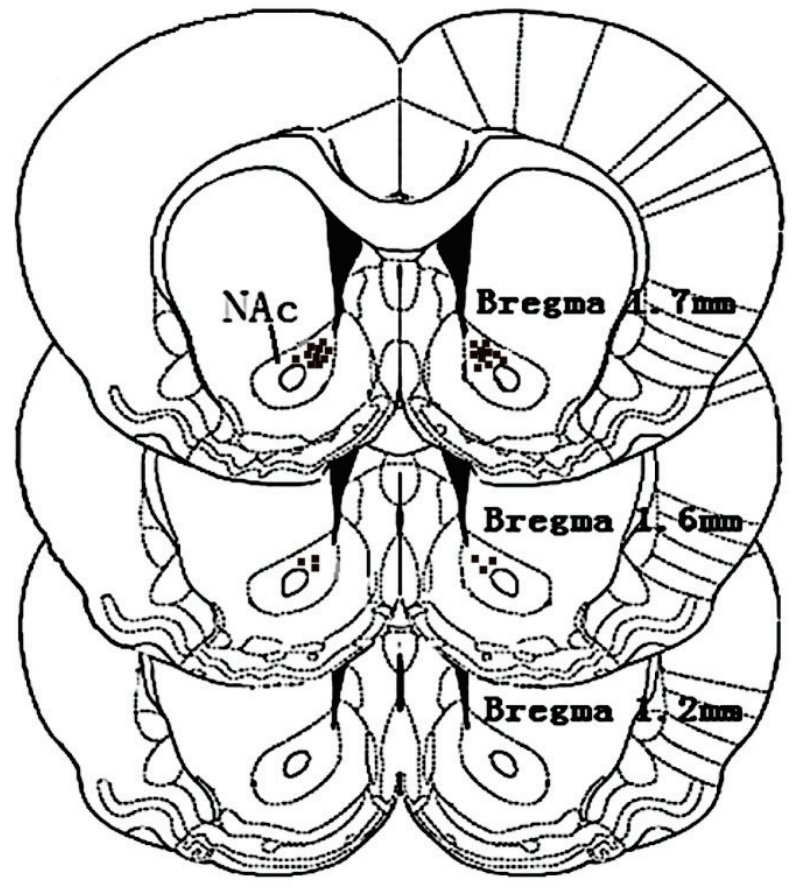

Fig. 1. Illustration of the location of the injection needle tips.

\section{Results}

Up-regulated p-PKA expression in the NAc in rats with mononeuropathy

At $7^{\text {th }}, 14^{\text {th }}$ and $28^{\text {th }}$ day after sciatic nerve ligation, the NAc tissues were excised, and Western blot was performed to test the expression of p-PKA. The results showed that the expression of p-PKA was significantly up-regulated at $14^{\text {th }}$ day $(\mathrm{n}=3 ; \mathrm{q}=7.930$; $\mathrm{P}<0.01)$ and $28^{\text {th }}$ day $(\mathrm{n}=3 ; \mathrm{q}=5.549 ; \mathrm{P}<0.05)$ to differ from the data of sham group, but the difference in the expression of $\mathrm{p}$-PKA was not significant at $7^{\text {th }}$ day $(\mathrm{n}=3$; $\mathrm{q}=3.701 ; \mathrm{P}>0.05$ ) after left sciatic nerve ligation, suggesting that the PKA signaling pathway may play a significant role in the transmission and modulation of neuropathic pain. The differences were determined by one-way ANOVA followed by Tukey's multiple comparison tests, as shown in Figure 2.

M617 down-regulated p-PKA expression, and M35 had no effect on $p-P K A$ expression in the NAc of neuropathic pain rats

Our studies demonstrated that M617 had an antinociceptive effect for neuropathic pain in the NAc (Duan et al. 2015). This study investigated the involvement of the PKA signaling pathway in the GalR1 activation-induced analgesic effect in the NAc of 


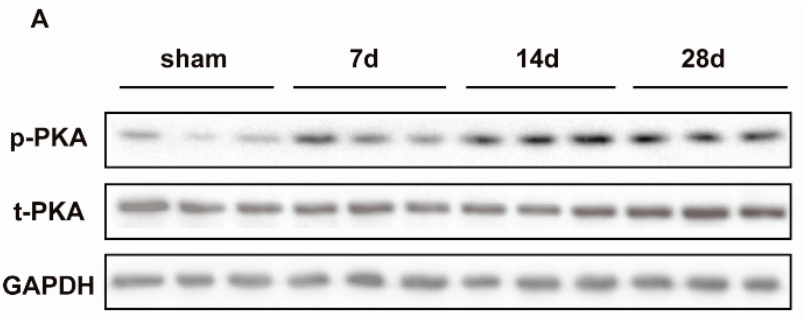

B

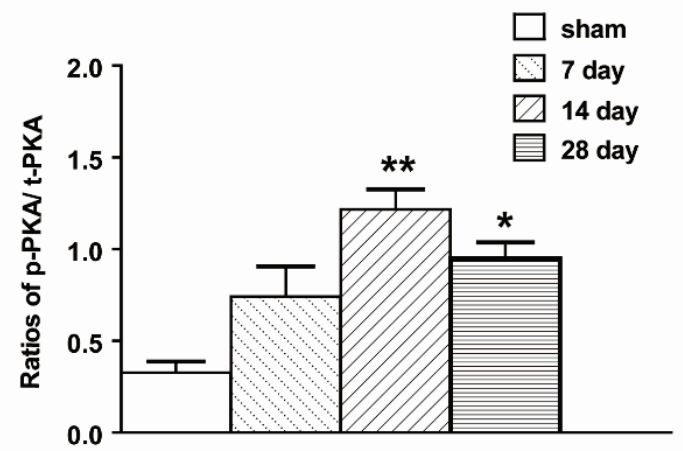

Fig. 2. The expression of p-PKA in the NAc in neuropathic pain rats. (A) Representative Western blots of p-PKA, t-PKA and GAPDH in the sham control group and left sciatic nerve-ligated group. (B) Histograms show the ratios of p-PKA/t-PKA. Data are presented as the mean \pm S.E.M. $* \mathrm{P}<0.05, * * \mathrm{P}<0.01$ compared with the sham group.

neuropathic pain rats. At $14^{\text {th }}$ day after sciatic nerve ligation, three groups of rats received an intra-NAc injection of $1 \mathrm{nmol} \mathrm{M} 617$ or $1 \mathrm{nmol} \mathrm{M} 35$ or $1 \mu \mathrm{l}$ saline, respectively. The NAc tissues were separated at $15 \mathrm{~min}$ after the injection, and the expression of p-PKA was examined by Western blot. As shown in Figure 3, the expression of p-PKA was significantly down-regulated after intra-NAc administration of M617 ( $\mathrm{n}=3, \mathrm{q}=6.817$, $\mathrm{P}<0.01$ ), whereas intra-NAc administration of M35 did not have a significant effect $(\mathrm{n}=3, \mathrm{q}=0.9851, \mathrm{P}>0.05)$. The differences were analysed by one-way ANOVA followed by Tukey's multiple comparison tests. The results showed that GalR1 plays an antinociceptive effect via inhibition of PKA activation in the NAc of rats with mononeuropathy, but blocking GalR1 had no effect on p-PKA expression, suggesting that there is other type of galanin receptor or signaling pathway related to antinociceptive effect via inhibiting PKA activation. M35 up-regulated p-PKA was counteracted by other type of galanin receptor activation or other signaling pathway down-regulated p-PKA.

M35 in the NAc blocked the M617-induced increase in the HWLs of rats with mononeuropathy, but M35 alone had no effect on HWLS

In order to study the effect of M35 further, seven

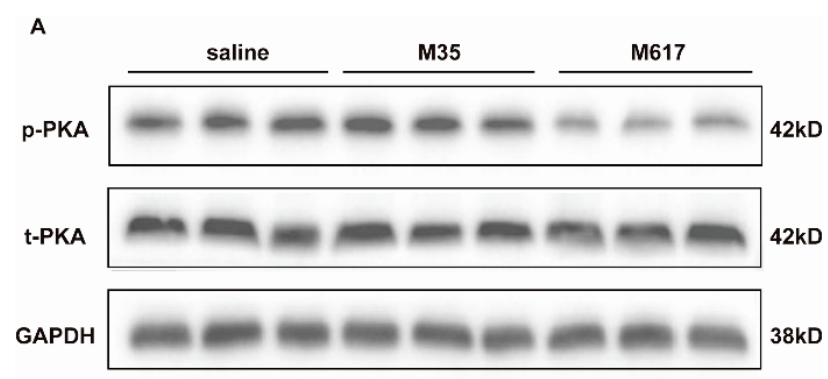

B

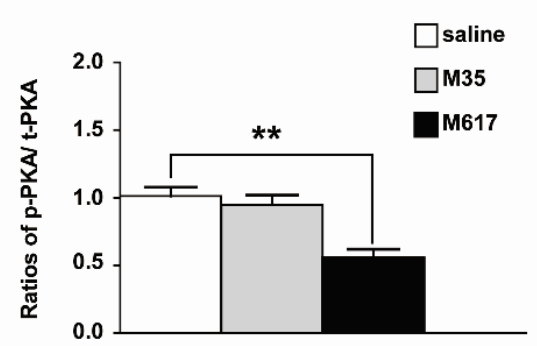

Fig. 3. Effects of M617 and M35 on p-PKA expression in the NAc of neuropathic pain rats. (A) Representative Western blots of p-PKA, t-PKA and GAPDH in the saline control group and intraNAC M617- and M35-injected groups. (B) Histograms show the ratios of $\mathrm{p}-\mathrm{PKA} / \mathrm{t}-\mathrm{PKA}$. Data are presented as the mean \pm S.E.M. $* * p<0.01$ compared with the saline group.

rats were given an intra-NAc injection of $1 \mu \mathrm{l}$ of $0.9 \%$ saline, followed $5 \mathrm{~min}$ later by intra-NAc injection of $1 \mathrm{nmol}$ of M35, as shown in Figure 4, M35 alone had no effect on HWLs. Furthermore, two groups of rats $(n=7)$ received intra-NAc injection of $1 \mathrm{nmol} \mathrm{M617}$, followed 5 min later by intra-NAc injection of $1 \mathrm{nmol}$ of M35 or saline. Compared to the HWLs in the saline control group, the M617-induced increases in the HWLs to thermal (left hind paw: $\mathrm{F}=40.59, \mathrm{P}<0.001$; right hind paw: $\mathrm{F}=23.79, \mathrm{P}<0.001$ ) and mechanical (left hind paw: $\mathrm{F}=69.91, \mathrm{P}<0.001$; right hind paw: $\mathrm{F}=45.63, \mathrm{P}<0.001$ ) stimulation were blocked by M35, as shown in Figure 4. The results showed that M35 in the NAc blocked the M617-induced an increase in the HWLs of rats with mononeuropathy, but M35 alone had no effect on HWLs, which is consistent with M35 had no effect on p-PKA expression. The difference was analysed by two-way repeated measures ANOVA followed by Bonferroni posttests.

PKA inhibitor H-89 increased HWLs and attenuated the M617-induced an increase in the HWLs in NAc of rats with mononeuropathy

In order to study the effect of intra-NAc injection of PKA inhibitor, seven rats were given an intra-NAc injection H-89 alone. As shown in Figure 5, compared with the HWLs of the DMSO control group 

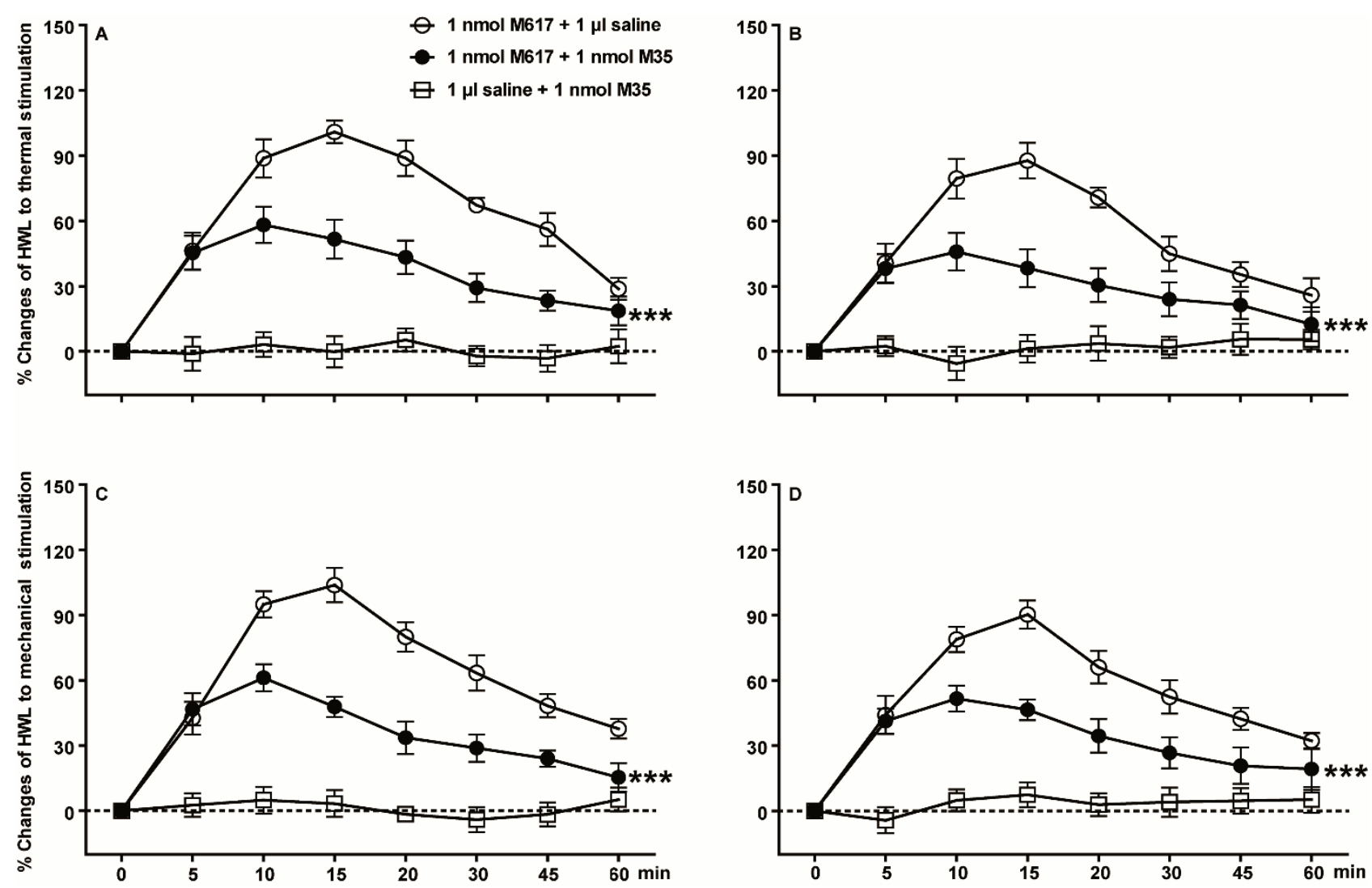

Fig. 4. Effects of intra-NAc injection of $M 35$ on the M617-induced antinociception in neuropathic pain rats. (A, B) \% change in HWL to thermal stimulation; (C, D) \% change in HWL to mechanical stimulation; A and C: Left HWL; B and D: right HWL. Time $=0$ min: intraNAc injection of $1 \mathrm{nmol}$ M617; time=5 min: intra-NAc injection of $1 \mathrm{nmol}$ M35 or $1 \mu \mathrm{l} 0.9 \%$ saline as the control group. Data are presented as the mean \pm S.E.M. $* * * P<0.001$, compared with the control group.

$(\mathrm{n}=7)$, the HWLs to thermal (left hind paw: $\mathrm{F}=52.86$, $\mathrm{P}<0.001$; right hind paw: $\mathrm{F}=31.24, \mathrm{P}<0.001)$ and mechanical (left hind paw: $\mathrm{F}=47.11, \mathrm{P}<0.001$; right hind paw: $\mathrm{F}=61.44, \mathrm{P}<0.001)$ stimulation were increased after intra-NAc injection $6 \mu \mathrm{g}$ of $\mathrm{H}-89$, the differences were analysed by repeated measures two-way ANOVA followed by Bonferroni post-tests. This result suggested that the PKA inhibitor H-89 in the NAc eased painrelated behaviour in rats with neuropathic pain.

To further demonstrate that PKA is a downstream signaling molecule of GalR1, we injected the GalR1 agonist M617 to activate GalR1 to induce increases in HWLs first, then we injected PKA inhibitor H-89 to test whether the antinociceptive effect of M617 would be attenuated injection of H-89. Four groups of rats received intra-NAc injection of $1 \mathrm{nmol}$ M617, followed 5 min later by intra-NAc injection of 1, 3, $6 \mu \mathrm{g}$ of the PKA inhibitor H-89 or DMSO as control group $(n=6)$. The differences were analysed by repeated measures two-way ANOVA followed by Bonferroni post-tests. As shown in Figure 5A and 5B, compared with the control group, the M617-induced increases in the HWLs to thermal stimulation were attenuated by intraNAc injection of $3 \mu \mathrm{g}$ (left hind paw: $\mathrm{F}=10.99, \mathrm{P}<0.01$; right hind paw: $\mathrm{F}=3.2, \mathrm{P}=0.10$ ) and $6 \mu \mathrm{g}$ (left hind paw: $\mathrm{F}=14.88, \mathrm{P}<0.01$; right hind paw: $\mathrm{F}=8.83, \mathrm{P}<0.05$ ), but not $1 \mu \mathrm{g} \mathrm{H}-89$ (left hind paw: $\mathrm{F}=2.94, \mathrm{P}=0.11$; right hind paw: $\mathrm{F}=0.18, \mathrm{P}=0.68$ ). In addition, as shown in Figure $5 \mathrm{C}$ and $5 \mathrm{D}$, intra-NAc injection of $3 \mu \mathrm{g}$ (left hind paw: $\mathrm{F}=9.96, \mathrm{P}<0.01$; right hind paw: $\mathrm{F}=5.94, \mathrm{P}<0.05$ ) and $6 \mu$ g (left hind paw: $\mathrm{F}=13.14, \mathrm{P}<0.01$; right hind paw: $\mathrm{F}=8.28, \mathrm{P}<0.05) \mathrm{H}-89$ dose-dependently attenuated the antinociceptive effect induced by M617 with mechanical stimulation, but there was a non-significant effect after injection of $1 \mu \mathrm{g} \mathrm{H}-89$ (left hind paw: $\mathrm{F}=1.79, \mathrm{P}=0.21$; right hind paw: $\mathrm{F}=0.94, \mathrm{P}=0.35$ ), suggesting that the $\mathrm{PKA}$ inhibitor H-89 attenuated the M617-induced an increase in the HWLs in NAc of rats with mononeuropathy. These results also showed that GalR1 plays an antinociceptive effect via inhibition of PKA activation in the NAc of rats with mononeuropathy. 

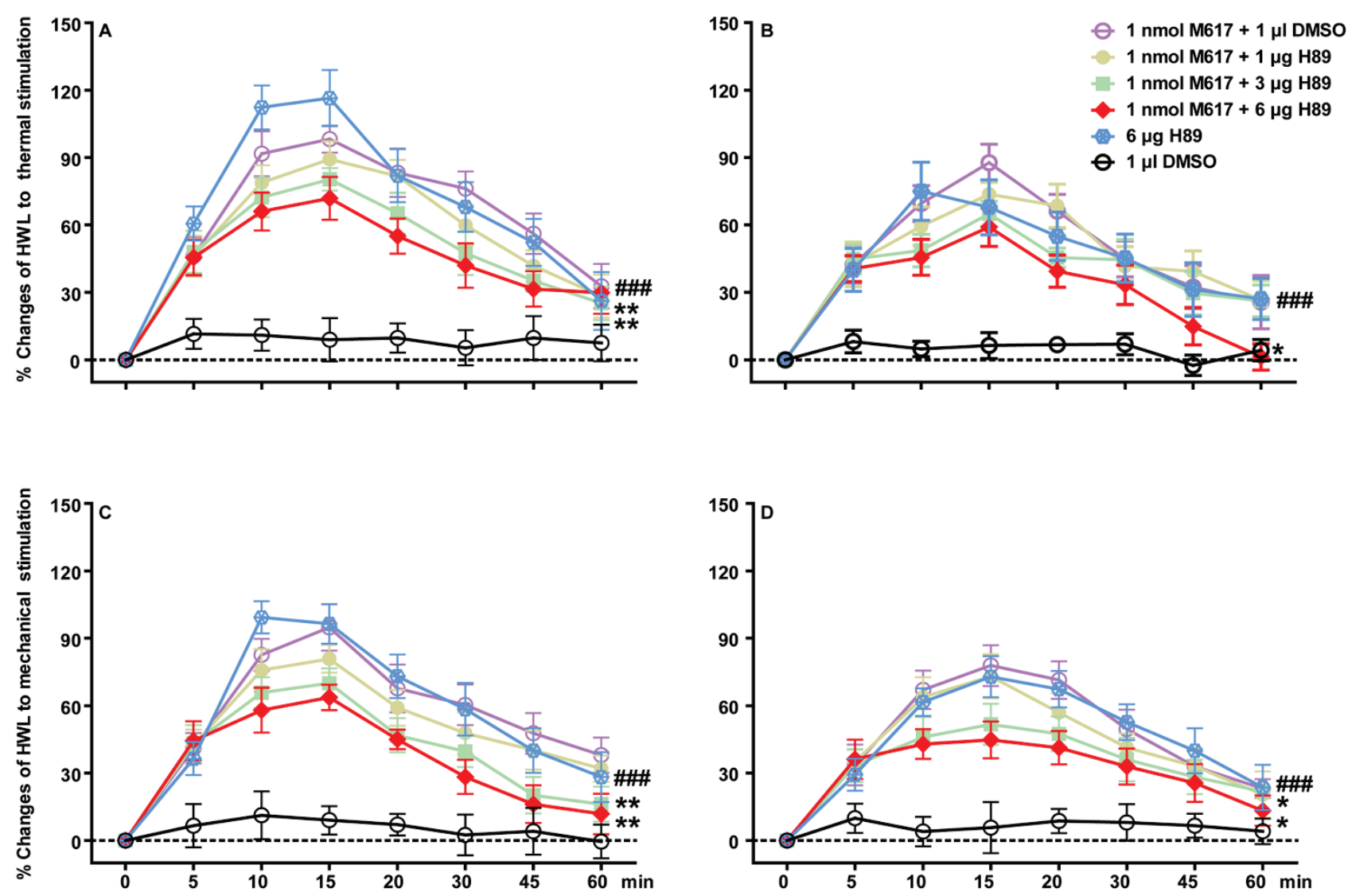

Fig. 5. Effects of intra-NAc injection of $\mathrm{H}-89$ on M617-induced antinociception in rats with neuropathic pain. NAc: nucleus accumbens; HWL: hind paw withdrawal latency. (A, B) \% changes of HWL to thermal stimulation; (C, D) \% changes of HWL to mechanical stimulation; A and C: Left HWL; B and D: right HWL. Data are presented as mean \pm S.E.M. $* P<0.05, * * P<0.01$, intra-NAC injection of

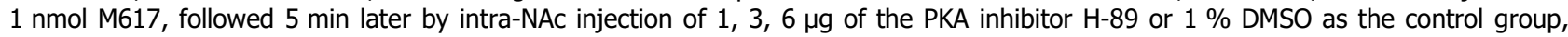
time $=0$ min: intra-NAc injection of $1 \mathrm{nmol}$ of M617, time $=5 \mathrm{~min}$ : intra-NAc injection of $1,3,6 \mu \mathrm{g}$ of $\mathrm{H}-89$ or $1 \mu \mathrm{l}$ of DMSO. \#\#\# P<0.001, intra-NAc injection $\mathrm{H}-89$ alone versus intra-NAc injection DMSO alone.

\section{Discussion}

Previous studies have shown that galanin is involved in the processing and development of nociceptive information in the CNS (Xu et al. 2012, Yang et al. 2015, Zhang et al. 2017). Galanin plays its roles via three GalRs: GalR1, -R2 and -R3, all of them are G-protein coupled receptors (Webling et al. 2012, Lang et al. 2015). Studies have proven that intra-central nucleus of the amygdala (Li et al. 2012) and intracerebroventricular (Fu et al. 2011) injection of M617, a GalR1-specific agonist (Lundström et al. 2005), induced an analgesic effect in intact rats. Our previous study also demonstrated that GalR1 expression was up-regulated, and M617 in the NAc induced an increase in the HWLs of rats with neuropathic pain (Duan et al. 2015), but its underlying mechanism is unclear.

PKA is widely distributed in the CNS. Some studies have demonstrated that the activation of the PKA signaling pathway is associated with the processing and development of nociceptive information. Intrathecal injection of the PKA inhibitor H-89 relieved the pain behaviour in rats with bone cancer-induced pain (Huang et al. 2012, Zhu et al. 2014). Additionally, electrophysiological experiment has also proven that the PKA signaling pathway was involved in modulating neuronal excitability in response to acute or chronic noxious stimulation in single DRG or spinal cord neurons (Huang et al. 2012). In the present study, the results showed that p-PKA expression in the NAc was up-regulated significantly at $14^{\text {th }}$ and $28^{\text {th }}$ day after sciatic nerve ligation in rats, suggesting an involvement of PKA in the transmission and modulation of neuropathic pain in the NAc of rats. Therefore, we wanted to know whether the PKA pathway was involved in the analgesic effect of GalR1 in the NAc of rats with neuropathic pain.

Interestingly, p-PKA expression was downregulated by intra-NAc administration of GalR1 agonist M617, whereas there was no significant difference in p-PKA expression after intra-NAc administration of M35, 
a general antagonist of GalR1 and GalR2 with an 18-fold selectivity for GalR1 over GalR2 in vitro (Webling et al. 2012). These results implied that GalR1 activation might play important roles in antinociception via PKA inhibition in the NAc of rats with mononeuropathy. In order to study this effect further, rats received intra-NAc injection of M617, followed $5 \mathrm{~min}$ later by intra-NAc injection of PKA inhibitor H-89, the results showed that H-89 attenuated the M617-induced an increase in the HWLs in NAc of rats with mononeuropathy, however intra-NAc injection of PKA inhibitor H-89 alone induced an increase in HWLs. Finally, we also found that M35 blocked the M617-induced an increase in the HWLs of rats with mononeuropathy, but M35 alone had no effect on HWL, these results suggested GalR1 plays an antinociceptive effect via inhibition of PKA activation in the NAc of rats with mononeuropathy, but may suggesting that there is other type of galanin receptor or other signal transduction pathway related to antinociceptive effect via inhibiting PKA activation.
In conclusion, this study showed that the involvement of PKA in neuropathic pain, GalR1 activation results in the inhibition of the PKA, leading to antinociception for neuropathic pain in the NAc of rats. This finding implied that GalR1 agonist could be potential and potent therapeutic option for neuropathic pain.

\section{Conflict of Interest}

There is no conflict of interest.

\section{Acknowledgements}

This study was supported by funds from the National Natural Science Foundation of China (NSFC 31360245), the Science and Technology Agency of Yunnan Province-Kunming Medical University Joint Special Foundation (2015FB012-) and the Science and Technology Agency of Yunnan Province-Third Affiliated Hospital of Yunnan Traditional Chinese Medical College (2017FF117-048).

\section{References}

BENNETT GJ, XIE YK: A peripheral mononeuropathy in rat that produces disorders of pain sensation like those seen in man. Pain 33: 87-107, 1988.

BRANCHEK TA, SMITH KE, GERALD C, WALKER MW: Galanin receptor subtypes. Trends Pharmacol Sci 21: 109-117, 2000.

COSTIGAN M, MOSS A, LATREMOLIERE A, JOHNSTON C, VERMA-GANDHU M, HERBERT TA, BARRETT L, BRENNER GJ, VARDEH D, WOOLF CJ, FITZGERALD M: T-cell infiltration and signalling in the adult dorsal spinal cord is a major contributor to neuropathic pain-like hypersensitivity. $J$ Neurosci 29: 14415-14422, 2009.

DUAN H, ZHANG Y, ZHANG XM, XU HH, SHU J, XU SL: Antinociceptive roles of galanin receptor 1 in nucleus accumbens of rats in a model of neuropathic pain. J Neurosci Res 93: 1542-1551, 2015.

DWORKIN RH, BACKONJA M, ROWBOTHAM MC, ALLEN RR, ARGOFF CR, BENNETT GJ, BUSHNELL MC, FARRAR JT, GALER BS, HAYTHORNTHWAITE JA, HEWITT DJ, LOESER JD, MAX MB, SALTARELLI M, SCHMADER KE, STEIN C, THOMPSON D, TURK DC, WALLACE MS, WATKINS LR, WEINSTEIN SM: Advances in neuropathic pain: diagnosis, mechanisms, and treatment recommendations. Arch Neurol 60: 1524-1534, 2003.

DWORKIN RH, TURK DC: Accelerating the development of improved analgesic treatments: the ACTION publicprivate partnership. Pain Med 12 (Suppl 3): S109-S117, 2011.

FU LB, WANG XB, JIAO S, WU X, YU LC: Antinociceptive effects of intracerebroventricular injection of the galanin receptor 1 agonist M617 in rats. Neurosci Lett 491: 174-176, 2011.

GEAR RW, LEVINE JD: Nucleus accumbens facilitates nociception. Exp Neurol 229: 502-506, 2011.

HANG LH, YANG JP, SHAO DH, CHEN Z, WANG H: Involvement of spinal PKA/CREB signalling pathway in the development of bone cancer pain. Pharmacol Rep 65: 710-716, 2013.

HANG LH, YANG JP, YIN W, WANG LN, GUO F, JI FH, SHAO DH, XU QN, WANG XY, ZUO JL: Activation of spinal TDAG8 and its downstream PKA signalling pathway contribute to bone cancer pain in rats. Eur J Neurosci 36: 2107-2117, 2012.

HO KY, SIAU C: Chronic pain management: therapy, drugs and needles. Ann Acad Med 38: 929-930, 2009. 
HUANG ZJ, LI HC, COWAN AA, LIU S, ZHANG YK, SONG XJ: Chronic compression or acute dissociation of dorsal root ganglion induces cAMP-dependent neuronal hyperexcitability through activation of PAR2. Pain 153: 1426-1437, 2012.

LANG R, GUNDLACH AL, HOLMES FE, HOBSON SA, WYNICK D, HÖKFELT T, KOFLER B: Physiology, signalling, and pharmacology of galanin peptides and receptors: three decades of emerging diversity. Pharmacol Rev 67: 118-175, 2015.

LI J, ZHANG JJ, XU SL, YU LC: Antinociceptive effects induced by injection of the galanin receptor 1 agonist M617 into central nucleus of amygdala in rats. Neurosci Lett 526: 45-48, 2012.

LUNDSTRÖM L, SOLLENBERG U, BREWER A, KOUYA PF, ZHENG K, XU X, SHENG X, ROBINSON JK, WIESENFELD-HALLIN Z, XU Z, HÖKFELT T, BARTFAI T, LANGEL L: A galanin receptor subtype 1 specific agonist. Int $J$ Pept Res Ther 11: 17-27, 2005.

SALGADO S, KAPLITT MG: The nucleus accumbens: A comprehensive review. Stereotact Funct Neurosurg 93: 75-93, 2015.

WEBLING KE, RUNESSON J, BARTFAI T, LANGEL U: Galanin receptors and ligands. Front Endocrinol (Lausanne) 3: 1-14, 2012.

WATANABE M, SUGIURA Y, SUGIYAMA E, NARITA M, NAVRATILOVA E, KONDO T, UCHIYAMA N, YAMANAKA A, KUZUMAKI N, PORRECA F, NARITA M: Extracellular N-acetylaspartylglutamate released in the nucleus accumbens modulates the pain sensation: Analysis using a microdialysis/mass spectrometry integrated system. Mol Pain 14: 1-10, 2018.

XU SL, LI J, ZHANG JJ, YU, LC: Antinociceptive effects of galanin in the nucleus accumbens of rats. Neurosci Lett 520: 43-46, 2012.

YANG Y, ZHANG Y, LI XH, LI Y, QIAN R, LI J, XU, SL: Involvements of galanin and its receptors in antinociception in nucleus accumbens of rats with inflammatory pain. Neurosci Res 97: 20-25, 2015.

ZHANG ML, WANG HB, FU FH, YU LC: Involvement of galanin and galanin receptor 2 in nociceptive modulation in anterior cingulate cortex of normal rats and rats with mononeuropathy. Sci Rep 7: 45930, 2017.

ZHU GQ, LIU S, HE, DD, LIU YP, SONG XJ: Activation of the cAMP-PKA signalling pathway in rat dorsal root ganglion and spinal cord contributes toward induction and maintenance of bone cancer pain. Behav Pharmacol 25: $267-276,2014$. 